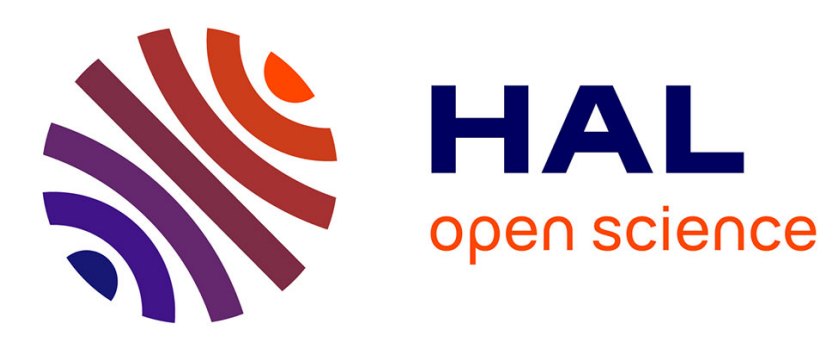

\title{
Les échanges gaziers entre la Russie et la Chine à l' aune de leur sécurité énergétique
}

Catherine Locatelli

\section{To cite this version:}

Catherine Locatelli. Les échanges gaziers entre la Russie et la Chine à l' aune de leur sécurité énergétique. Revue d'Etudes Comparatives Est-Ouest, 2015, 46 (02), pp.83-104. 10.4074/S033805991500203X . hal-01206226

\section{HAL Id: hal-01206226 \\ https://hal.univ-grenoble-alpes.fr/hal-01206226}

Submitted on 28 Sep 2015

HAL is a multi-disciplinary open access archive for the deposit and dissemination of scientific research documents, whether they are published or not. The documents may come from teaching and research institutions in France or abroad, or from public or private research centers.
L'archive ouverte pluridisciplinaire HAL, est destinée au dépôt et à la diffusion de documents scientifiques de niveau recherche, publiés ou non, émanant des établissements d'enseignement et de recherche français ou étrangers, des laboratoires publics ou privés. 


\section{ÉCONOMIE DU DÉVELOPPEMENT DURABLE ET DE L'ÉNERGIE}

\section{Les échanges gaziers \\ entre la Russie et la Chine \\ à l'aune de leur sécurité énergétique}

\section{Catherine Locatelli}

février 2015

Cahier de recherche EDDEN n 2/2015

UMR PACTE - pôle EDDEN

BP 47 - 38040 Grenoble CEDEX 9 - France

1221 rue des Résidences - 38400 Saint Martin d'Hères

P a c t e Tél.: +33(0)4 76825692 -Télécopie : +33 (0)4 56528571

http://edden.upmf-grenoble.fr 



\title{
Les échanges gaziers entre la R ussie et la C hine à l'aune de leur sécurité énergétique
}

\author{
C. Locatelli $i^{1,2}$ \\ ${ }^{1}$ Univ. Grenoble A Ipes, PACTE, EDDEN, F-38000 Grenoble, France \\ ${ }^{2}$ CNRS, PACTE, EDDEN, F-38000 Grenoble, France
}

Février 2015

L'accord signé entre la compagnie russe Gazprom et la compagnie chinoise CNPC en mai 2014, relatif à l'exportation de $38 \mathrm{G} \mathrm{m}^{3}$ /an pendant 30 ans de gaz russe vers la Chine, marque un tournant dans la politique énergétique de la Russie comme dans celle de la Chine. Les volumes concernés sont faibles, mais ils n'enlèvent rien à la portée économique et stratégique de l'accord qui apparaît logique compte tenu de la proximité des marchés et des complémentarités existant entre les deux pays. D'un côté, la Chine est susceptible à moyen terme de s'affirmer comme un importateur majeur de gaz naturel compte tenu des perspectives de croissance de sa demande. De l'autre, la R ussie possède d'immenses réserves gazières en Sibérie orientale et en Extrême-Orient qu'elle entend mettre en production, d'abord dans la perspective de développer économiquement ces régions (Shadrina \& B rashaw, 2013).

Pour les deux parties, ces échanges gaziers font partie d'une stratégie de diversification qui vise à répondre à des préoccupations de sécurité énergétique dans ses deux dimensions, offre et demande. II s'agit pour la Chine de sécuriser son approvisionnement gazier par la diversification de ses fournisseurs et de ses routes d'importation (sécurité de l'offre). II s'agit pour la Russie de diversifier ses marchés d'exportation pour sécuriser la demande gazière qui lui sera adressée. II apparaît ainsi clairement que la stratégie de diversification des marchés d'exportation menée par Gazprom et soutenue par l'Etat russe s'affirme comme la réponse apportée par l'entreprise aux incertitudes de son principal marché d'exportation, I'UE. Sa stratégie « asiatique » ne peut ainsi s' analyser sans référence aux évolutions du marché gazier européen.

Le développement des échanges gaziers entre la Russie et la Chine s'inscrit dans un contexte institutionnel particulier. Ce dernier est marqué par une relative proximité des modèles d'organisation de leurs industries gazières respectives, ainsi que des modalités de leur régulation (règles, normes). Ainsi, au modèle concurrentiel d'organisation des industries gazières promu par l'UE répond le modèle plus hiérarchique de la R ussie et de la Chine centré sur des compagnies d'E tat, une intégration verticale, et des marchés que l'on peut qualifier de duaux. On peut dès lors faire I'hypothèse que les « différences institutionnelles » évoquées à propos des échanges gaziers entre la Russie et l'UE sont moins prégnantes entre la Chine et la Russie (Shadrina, 2014a; Romanova, 2014 ; Locatelli, 2013). Ces spécificités ont leur traduction dans les logiques de gestion de la sécurité énergétique. Au cadre multilatéral et concurrentiel de l'UE s' oppose celui des relations bilatérales et de la diplomatie énergétique mis en œuvre par la R ussie et la Chine. 
L'objet de cet article est d'analyser le développement des échanges gaziers entre la Chine et la Russie en tant que réponse apportée à leurs enjeux de sécurité énergétique. L'hypothèse développée est que ces échanges sont portés par des arrangements institutionnels relativement cohérents et complémentaires (au sens défini par A oki, 2001). Ainsi la réorientation des exportations gazières de la Russie vers la Chine, même si elle n'en est qu'à ses débuts, se fonde sur une « convergence institutionnelle » de son industrie gazière avec celle de la Chine, alors que de profondes divergences institutionnelles marquent les relations entre l'UE et la Russie. Cet article s'inscrit dans les approches multiniveaux développées par l'analyse néoinstitutionnelle (Williamson, 2000 ; Dixit, 2009) et les approches portant sur les questions d'alignement entre la structure de gouvernance et l'environnement institutionnel (Williamson, 2005 ; B rousseau et al., 2011).

\section{I - L'Asie dans la stratégie de sécurisation de la R ussie}

Les exportations gazières de la Russie vers I'A sie (Japon, Corée du Sud, Chine) s'inscrivent dans une politique de diversification des marchés qui répond à une volonté de sécurisation de la demande. Elle est la réponse stratégique apportée par Gazprom et la R ussie aux incertitudes du marché gazier européen. L'UE, avec $163 \mathrm{Gm}^{3}$ de gaz russe importés en 2013, demeure le marché privilégié d'exportation de Gazprom pour lequel la compagnie entend rester un fournisseur fiable et significatif. $M$ ais divers facteurs sont venus modifier les équilibres établis depuis les années 1970. D'une part, les évolutions structurelles du marché de l'UE - faible augmentation de la demande à moyen terme, marché plus concurrentiel en raison des politiques de libéralisation de l'UE, impact des shale gas - ont induit un environnement plus incertain pour la compagnie gazière russe. Elles imposent à Gazprom de redéfinir ses stratégies contractuelles tant en termes de prix que de volume. D'autre part, la relation gazière entre l'UE et la Russie est marquée depuis le début des années 2000 par des incompréhensions et des conflits croissants (B oussena \& Locatelli, 2013). La question de la sécurité est ainsi redevenue une thématique importante de ces relations.

La préoccupation des autorités chinoises porte autant sur les risques d'une rupture physique de l'approvisionnement que sur les conséquences d'un renchérissement des prix énergétiques mondiaux sur son économie, susceptible d'handicaper la compétitivité de ses exportations. La sécurité énergétique est devenue un objectif essentiel de la politique énergétique de la Chine, laquelle s' affirme comme un importateur d'hydrocarbures majeur.

\subsection{L es objectifs de la R ussie vis-à-vis de l'A sie}

La volonté de l'Etat russe de s'ouvrir de nouveaux marchés d'exportation vers I'A sie date de la fin des années 1990 et transparaît clairement dans sa Stratégie E nergétique de long terme de 2020 et 2030. Celle-ci table en effet sur des exportations gazières à destination de cette zone de l'ordre de $75 \mathrm{G} \mathrm{m}^{3}$ en 2030, I'A sie devant représenter 19-20\% des exportations gazières du pays. Le volume des réserves de la Sibérie orientale et de l'Extrême-Orient permet d'envisager un développement important de la production gazière à l'Est du pays. Selon les données de Gazprom, les réserves prouvées de cette zone se monteraient à 5 trilliards de $\mathrm{m}^{3}$ (Gazprom, 2013). 


\section{Tableau 1 : L'A sie dans la Stratégie E nergétique de long terme de la R ussie dans le domaine gazier}

\begin{tabular}{|l|c|c|c|}
\hline & Phase 1: 2013-15 & Phase 2: 2016-20/22 & Phase 3 : 2021/23-30 \\
\hline Sibérie orientale, $\mathrm{Gm}^{3}$ & $9-13$ & $26-55$ & $45-65$ \\
\hline Extrême-Orient, G m & $34-40$ & $65-67$ & $85-87$ \\
\hline $\begin{array}{l}\text { Part de I'A sie dans les } \\
\text { exportations en \% }\end{array}$ & $11-12$ & $16-17$ & $19-20$ \\
\hline $\begin{array}{l}\text { Part des exportations de } \\
\text { GNL en \% }\end{array}$ & $4-5$ & $10-11$ & $14-15$ \\
\hline
\end{tabular}

Source : Energy Strategy of Russia for the period up to 2030. Ministry of Energy of The Russian Federation, M oscow, 2010.

Cette stratégie d' exportation à destination de l'A sie présente deux spécificités. D'une part, les nombreux projets évoqués se déclinent en deux options, l'une consistant en des exportations de GNL, l'autre en des exportations par gazoduc (cf. tableau 2). D' autre part, ils ne sont pas tous portés par Gazprom mais relèvent d'acteurs assez différenciés, comme la compagnie pétrolière d'Etat Rosneft, ou «d'indépendants » comme la compagnie gazière privée Novatek. Esquissant un début de concurrence entre les acteurs russes quant à I'approvisionnement de l'A sie, cette politique témoigne d'une rupture assez marquée dans la logique d'exportation gazière de la Russie si l'on se réfère à la stratégie menée en Europe dominée par Gazprom et un approvisionnement par gazoduc. A partir de 2013, le monopole sur l'ensemble des exportations que détenait Gazprom (depuis le début des années 2000) est désormais restreint aux seules exportations par gazoduc. Les conditions sous lesquelles sont autorisées les exportations de GNL par d'autres acteurs restent cependant extrêmement restrictives puisque aujourd'hui seuls Rosneft, Novatek et Gazprom répondent aux critères exigés pour permettre des exportations de GNL vers l'A sie $^{1}$. La politique mise en place vise également à dével opper une stratégie de marchés alternatifs J apon, Chine, Corée du Sud, afin d'échapper à la dépendance vis-à-vis d'un seul pays.

\section{Tableau 2 : Principaux projets de G NL et de gazoducs de la $R$ ussie à destination de l'A sie}

\begin{tabular}{|c|c|c|c|c|c|}
\hline Projets & $\begin{array}{l}\text { Principaux } \\
\text { acteurs }\end{array}$ & Gisements & Capacité & M ise en service & M archés visés \\
\hline \multicolumn{6}{|l|}{ GNL } \\
\hline $\begin{array}{l}\text { V ladivostok } \\
\text { LNG }\end{array}$ & Gazprom & $\begin{array}{l}\text { Sakhaline 3, } \\
\text { K ovytkta, } \\
\text { Chayanda }\end{array}$ & $\begin{array}{l}3 \text { trains de } 5 \\
\text { M t/an }\end{array}$ & $\begin{array}{l}1 \text { train : } 2018 \\
2 \text { trains : } 2020 \\
3 \text { trains : ? }\end{array}$ & A sie dont J apon \\
\hline Y amal LNG & $\begin{array}{l}\text { Novatek }(60 \%), \\
\text { Total }(20 \%), \\
\text { CNPC }\end{array}$ & $\begin{array}{l}\text { Y uzhno- } \\
\text { Tambey } \\
\text { (Y amal) }\end{array}$ & 16,5 M t/an & $\begin{array}{l}1 \text { train : } 2017 \\
2 \text { trains : } 2018 \\
3 \text { trains : } 2019\end{array}$ & Europe et A sie \\
\hline $\begin{array}{l}\text { Projet de GNL } \\
\text { dans la } \\
\text { péninsule de } \\
\text { Gydan }\end{array}$ & N ovatek & $\begin{array}{l}\text { Salmanovsk et } \\
\text { Geofizik }\end{array}$ & & & Asie \\
\hline Sakhaline 1 & $\begin{array}{l}\text { Rosneft- } \\
\text { ExxonM obil }\end{array}$ & $\begin{array}{l}\text { Sakhaline I } \\
\text { M er d'Okhotsk }\end{array}$ & $5 \mathrm{M} \mathrm{t} / \mathrm{an}$ & 1 train : 2018 & A sie-Pacifique \\
\hline Sakhaline 2 (1) & Gazprom-Shell & $\begin{array}{l}\text { Sakhaline } 2 \text { ou } \\
\text { Sakhaline } 3\end{array}$ & $5 \mathrm{Mt}$ tan & 1 train : 2018 & Chine \\
\hline
\end{tabular}

\footnotetext{
${ }^{1}$ Selon la loi de décembre 2013, les compagnies pouvant obtenir des droits d'exportation en matière de GNL sont soit des compagnies détenues à plus de $50 \%$ par l'Etat russe, exploitant des gisements offshore, soit des compagnies détentrices de ressources naturelles dont la production est destinée à être liquéfiée ou ayant une licence pour construire une usine de GNL (M itrova, 2014).
} 


\begin{tabular}{|l|l|l|l|l|l|}
\hline Gazoducs & & & & & \\
\hline $\begin{array}{l}\text { Power of } \\
\text { Siberia }\end{array}$ & $\begin{array}{l}\text { Gazprom- } \\
\text { CNPC }\end{array}$ & Chayandinskoye & $38 \mathrm{Gm}^{3}$ & 2018 & Chine \\
\hline Altaï & Gazprom & $\begin{array}{l}\text { Gisements de } \\
\text { Sibérie } \\
\text { occidentale }\end{array}$ & & & \\
\hline
\end{tabular}

Note (1) : Sakhaline produit déjà 10,8 M t/an de GNL exporté en A sie, en particulier au Japon.

Sources : Russia pays high price for export prize, Petroleum E conomist, October 2012; Russia reviews LNG export policy, International Gas Report, n 718, 25/02/2013 ; IEA (2014), Russia 2014, OECD/IEA.

A ux conditions actuelles, tous les projets d'exportation vers l'A sie envisagés ne sont pas économiquement viables. Toutefois, la politique d'exemption fiscale mise en œuvre par le gouvernement pour le développement de certains gisements de Sibérie orientale et certains projets d'exportation améliorent grandement leur rentabilité, à l'image du Y amal LNG (Henderson \& Stern, 2014). Ces exemptions portent sur la M ineral Extraction Tax et celle relative aux exportations. Elles démontrent combien cette politique d'exportation vers I'A sie est stratégique pour l'Etat russe.

Elément important de la justification économique de ces projets, les exportations de gaz naturel vers l'A sie s'inscrivent dans un programme plus large de "gazéification » de la Sibérie orientale et de l'Extrême-Orient. L'Eastern gas program, adopté en 2007, vise à développer de manière coordonnée et unifiée un système de production et de transport (gazoducs) dans cette zone. La création de quatre centres de production est prévue. Le centre de Y akutsk est basé sur le développement du gisement de Chayandiskoye qui, devant être rapidement dével oppé par Gazprom, pourrait alimenter le gazoduc Power of Siberia. A celuici s'ajoutent le centre d'Irkustk (gisement de Kovytka), celui de Krasnoyarsk et celui de Sakhaline (Paik, 2012).

\subsection{L'A sie : la réponse aux incertitudes du marché gazier européen dans la stratégie de Gazprom}

La réorientation des exportations gazières vers I'A sie ne peut être qu' une stratégie de moyenlong terme puisque, sel on J. Henderson et J. Stern (2014), à I'horizon 2025 les exportations de la Russie à destination de l'A sie pourraient être, dans un scénario optimiste, de l'ordre de $100 \mathrm{Gm}^{3}$. M ais elle s'inscrit dans une logique de sécurisation des marchés et de la demande. Elle est la réponse de la R ussie et de sa compagnie gazière aux incertitudes du marché gazier de l'UE. Elle peut permettre à Gazprom de compenser les éventuelles pertes de revenus sur le marché européen liées à un environnement plus concurrentiel, et par conséquent pour l'Etat russe de compenser des rentrées fiscales moindres. De manière plus générale, la crédibilité d'exportations significatives en A sie est un facteur essentiel dans l'appréciation que I'on peut porter sur le comportement possible de Gazprom par rapport à une concurrence accrue sur le marché européen.

Le marché de I'UE a de tout temps été de première importance pour l'Etat russe et pour Gazprom, qui cherche sur ce marché à maximiser ses revenus ${ }^{2}$ (en termes de prix et de volume). Les exportations vers cette zone remplissent trois principales fonctions: assurer la rentabilité de la compagnie gazière (compte tenu des bas prix du gaz en interne mais aussi, dans les années 1990, des phénomènes de troc et de non-paiement, cf. Locatelli, 2014) ; permettre un approvisionnement stable en gaz à l'économie russe, les ventes de gaz naturel

\footnotetext{
${ }^{2}$ J. Stern (2014) qualifie ainsi le comportement de Gazprom de monopole maximisateur.
} 
sur le marché gazier européen subventionnant les consommateurs russes sous la forme de prix réglementés relativement faibles; enfin, elles assurent à l'Etat des rentrées fiscales importantes, les hydrocarbures étant des variables essentielles de la stabilité du budget de I'Etat et de la croissance économique du pays. Ce type de comportement visant la recherche d'une maximisation des revenus est aujourd'hui mis en cause par les évolutions du marché européen. II est ainsi significatif que, sur la période 2008-2012, la part des ventes à l'UE dans les revenus de Gazprom soit en diminution au profit des ventes sur le marché intérieur russe 3 . M ême si le marché gazier européen continue à assurer une grande partie de ses revenus à Gazprom, ces évolutions traduisent des changements de rentabilité entre les deux marchés.

La faible croissance de la demande gazière européenne et le processus de libéralisation qui résulte en un marché plus concurrentiel soumis à de nouvelles règles d'échanges supposent de la part des fournisseurs traditionnels de I'UE des adaptations contractuelles importantes, sous peine de voir leur part de marché diminuer significativement. Les fournisseurs traditionnels de I'UE se trouvent contraints dans leur stratégie d'exportation à redéfinir l'arbitrage jusque-là effectué entre volume et prix, notamment dans les contrats de long terme de type Take or Pay (contrats TOP). Ceux-ci sont la forme contractuelle sous laquelle est organisée la fourniture gazière russe à l'UE. Ils lui garantissent sur le long terme la demande, ce qui aux yeux de la compagnie est la condition du développement de nouvelles zones de production en raison de l'importance des investissements à réal iser.

Tout d'abord, le processus de libéralisation des industries gazières de I'UE induit des modifications substantielles de certaines clauses des contrats de long terme Take or Pay, souvent mis en cause au nom des principes concurrentiels (Hautecloque \& Glachant, 2011; Percebois, 2008) $)^{5}$. Ensuite, la clause d'indexation des prix du gaz sur ceux du pétrole et des produits pétroliers, à laquelle Gazprom est particulièrement attachée, est mise en question par les évolutions du marché gazier européen. Alors que les prix des contrats TOP ont suivi l'évolution des prix du pétrole, en 2009-2010, les prix du gaz naturel et du GNL vendus sur les marchés spot se sont effondrés du fait de la surabondance de gaz. II en a résulté des baisses significatives des cours sur les marchés spot et un découplage important entre les prix spot et les prix des contrats de long terme. Cette évolution a conduit la plupart des compagnies gazières européennes à demander une révision de leur contrat de long terme, notamment ceux signés avec Gazprom ${ }^{6}$. Face à des pertes de marché significatives ${ }^{7}$, la compagnie gazière a

\footnotetext{
${ }^{3}$ Sur la période 2008-2012, la part des ventes à l'UE dans les revenus de Gazprom est passée de 60 à $53 \%$, celle à la CEI de 17 à $29 \%$, celle à la Russie de 23 à $29 \%$ (Henderson \& Pirani, 2014).

${ }^{4}$ Selon les données fournies par Gazprom (2011), le prix moyen de ventes de gaz naturel sur le marché russe était de $77 \$ / 1000 \mathrm{~m}^{3}$ en 2010 contre une moyenne de $244 \$ / 1000 \mathrm{~m}^{3}$ sur le marché européen. II serait en moyenne aujourd' hui pour les consommateurs industriels de l'ordre de $120 \$ / 1000 \mathrm{~m}^{3}$ (Gazprom, A nnual report 2013).

${ }^{5}$ Pour une analyse détaillée du débat, on pourra se reporter à L ocatelli (2013).

${ }^{6}$ Depuis lors se développe un débat croissant sur le futur et la pertinence de maintenir dans les contrats de long terme l'indexation des prix du gaz sur ceux des produits pétroliers, comme l'illustre la controverse entre d'une part S. Komlev, de la Direction des prix et des contrats de la société d'exportation de Gazprom, partisan de I'indexation sur les prix du pétrole, et d'autre part J. Stern et $\mathrm{H}$. Rogers, deux chercheurs de l'Oxford Institute for Energy Studies, qui s'y opposent (K omlev, 2013 ; Stern \& Rogers, 2013).
}

${ }^{7}$ En 2012, ses exportations gazières à destination de I'UE diminuent de $5 \%$ alors que dans le même temps, celles de la N orvège augmentent. 
choisi à partir de 2012 d'ajuster ses prix à la baisse, afin de préserver une certaine compétitivité. La baisse des prix s'opère selon deux logiques. D'une part, la compagnie diminue le prix dans la formule d'indexation prix de base ${ }^{8}$. D'autre part, Gazprom accorde à un certain nombre de ses clients des rabais estimés, dans la littérature consacrée à ce sujet, à $10-20 \%{ }^{9}$. En conséquence, en 2013 , le prix du gaz russe a été en moyenne de $387 \$ / 1000 \mathrm{~m}^{3}$ (soit $10 \$ / M B T U)^{10}$. Dans ces conditions, Gazprom sera d'autant plus susceptible, et en mesure, de jouer la logique concurrentielle du marché gazier européen qu'il disposera d'autres débouchés, notamment en A sie.

A u-delà de la question des prix, de nombreuses régulations du marché gazier européen restent problématiques pour Gazprom. L'obligation de se conformer aux règles d'accès aux tiers pour la construction de nouveaux gazoducs (cf. le gazoduc South Stream), ou encore les difficultés croissantes à poursuivre une politique d'intégration vers l'aval du marché européen en raison de la clause du pays tiers, sont au nombre de celles-ci. Globalement, la nouvelle architecture du marché gazier telle qu'esquissée dans le Gas Target Model avec un système d'EntréeSortie est susceptible de déboucher sur des phénomènes de discordance contractuelle (contractual mismatches), dès lors que la durée des contrats d'approvisionnement serait de plus long terme que les contrats de réservations de capacité de transport (Talus, 2011; K onoplyanik, 2005). Cette question est particulièrement épineuse pour Gazprom qui serait dans l'obligation, pour fournir ses clients, de procéder par appel d'offre à la réservation de capacités sur différentes zones d'Entrée-Sortie (Y afimava, 2013). Le risque de multiples contractual mismatches ne peut être exclu, introduisant un risque spécifique sur la fourniture gazière de l'UE en provenance de l'extérieur (Boltz \& Konoplyanik, 2012 ; 2013).

\section{II - La Russie dans la politique de sécurisation des approvisionnements énergétiques dela Chine}

La logique de sécurisation par la diversification des marchés s'accorde à la politique de diversification des sources et des routes (gazoduc versus GNL) d'approvisionnement menée par la Chine, notamment au travers de ses compagnies d'Etat. Partie prenante de la politique de sécurisation de l'offre menée par ce pays face à la croissance prévisible de ses besoins en gaz naturel, elle est un des axes structurants de la stratégie énergétique chinoise.

\subsection{La politique de diversification des sources et des voies d'approvisionnement de la Chine}

A u travers de sa fourniture contractuelle, la Chine dispose aujourd'hui de quatre grandes sources d'approvisionnement en gaz naturel, avec des d'importations par gazoducs et des importations de GNL. II s'agit d'abord d'importations en GNL (25 Gm ${ }^{3}$ en 2013) dont la fourniture est dominée par le Q atar (38 \% de la fourniture de GNL chinois) et prochainement l'A ustralie. Pour les 20-30 prochaines années, les différents contrats passés (fin 2014) lui assurent une fourniture de l'ordre de $70 \mathrm{Gm}^{3}$ en matière de $\mathrm{GNL}$. La fourniture par gazoduc $\left(27,4 \mathrm{G} \mathrm{m}^{3}\right.$ en 2013$)$ est assurée par trois grandes sources, les importations en provenance de

\footnotetext{
${ }^{8} \mathrm{~J}$. Stern (2014). Sel on lui, la baisse du prix de base dans la formule d'indexation aurait été de l'ordre de 7-10\%.

${ }^{9}$ Ces rabais sont fonction des différences de prix entre les contrats de long terme et les marchés spot. En 2012, la compagnie aurait versé plus de 3 milliards de dollars à différentes compagnies européennes.

${ }^{10}$ Uncomfortable bedfellows, Petroleum E conomist, J une 2014.
} 
Myanmar, celles d'A sie centrale (Turkménistan, Kazakhstan et Ouzbékistan) et prochainement celles en provenance de Russie. Le gazoduc A sie central e-Chine dispose d'une capacité de $55 \mathrm{Gm}^{3}$ /an qui en 2020 pourrait être portée à $85 \mathrm{Gm}^{3}$, celui de Myanmar de $12 \mathrm{G} \mathrm{m}^{3} /$ an. Enfin, le Power of Siberia en provenance de Russie permettra d'ici 2020 d'ajouter $38 \mathrm{Gm}^{3} / a n$ à la fourniture chinoise.

- L'importance de l'accord Russie-Chine : une fourniture concurrentielle

Le contrat signé entre les deux partenaires est un contrat de long terme (30 ans) de type Take or Pay conformément à l'essentiel de l'approvisionnement gazier chinois, la Chine pouvant toutefois ponctuellement se fournir sur le marché spot. Signé en mai 2014 entre Gazprom et la CNPC, il porte sur une fourniture de $38 \mathrm{Gm}^{3} / a n$ à partir de 2018 , quantité qui pourrait être portée à $60 \mathrm{Gm}^{3}$ (cf. tableau 2). La val eur du contrat est de 400 milliards de dollars sur 30 ans.

La question du prix des exportations gazières russes dans ce contrat est un facteur essentiel à analyser, notamment dans sa comparaison avec les autres contrats signés par la Chine, mais aussi par rapport à ceux signés par Gazprom en Europe. Durant de nombreuses années sa signature a buté plus spécifiquement sur cette question des prix. Gazprom considérait les prix européens comme la référence pour l'ensemble de ses exportations ${ }^{11}$, alors que la Chine faisait du contrat signé avec le Turkménistan le point de référence de son approvisionnement par gazoduc, mettant ainsi les fournisseurs en concurrence. Pékin entend en effet disposer d'un approvisionnement sécurisé en termes de quantité mais aussi à un coût «acceptable ». M ême si nous ne disposons que de données fragmentaires, les termes du contrat étant confidentiels, sa valeur donne une première indication de ce que pourrait être le prix du gaz exporté vers la Chine. II pourrait ainsi se situer autour de 10 \$/M BTU selon de nombreux experts (Cornot-Gandol phe, 2014 ; Henderson \& Stern, 2014).

Ces niveaux de prix sont susceptibles de répondre aux intérêts chinois et russes (Henderson \& Stern, 2014). Ils assurent un taux de rentabilité suffisant pour G azprom, et pour la CN PC sont du même ordre de grandeur que ceux de ses importations en provenance d'A sie centrale). On peut considérer, comme le fait J. Henderson (2014), que la politique de sécurisation de la Chine passe par le maintien d'un équilibre concurrentiel entre les quatre grandes sources d'approvisionnement dont elle dispose actuellement. Dès lors, le prix du contrat russe est susceptible de servir de benchmarking pour les autres sources d'approvisionnement futures, en particulier celles de GNL. Du côté de la Russie, ce niveau de prix est proche de celui des contrats de long terme de Gazprom à la frontière allemande. Compte tenu des révisions de prix de ces contrats, il s' est établi à 9,15 \$/M BTU début 2015. Par ailleurs, la baisse des prix du pétrole est susceptible d'impacter fortement ces prix, compte tenu des formules d'indexation des contrats de long terme.

\section{Encadré 1 : E stimation du prix des livraisons gazières à la C hine (au point d'entrée à la frontière), premier semestre 2014}

- Prix moyen des importations de gaz turkmène : 10,4 \$/M BTU (M ais selon J. Henderson (2014) il faut rajouter 4,48 \$/M BTU de coût de transport jusqu' à Shanghai contre seulement 2,50 \$/M BTU pour le gaz russe en provenance du Power of Siberia)

- Prix moyen des importations de gaz M yanmar (par gazoduc) : 11,6 \$/M BTU

- Prix estimé du gaz russe : 10 \$/M BTU

\footnotetext{
${ }^{11}$ Pour une analyse de ces négociations, on pourra se reporter à Henderson (2011) et Paik (2012).
} 
- Prix moyen des importations de GNL : 12,6 \$/M BTU (M ais certains GNL en provenance du M oyenOrient ou d'Afrique peuvent atteindre 17 à 19 \$/MBTU. Ainsi, le prix du gaz qatari, dont I'approvisionnement en volume est important pour la Chine, aurait été en moyenne de 17,30 \$/M BTU en 2013)

Sources: Russia, China sign historic agreement, International Gas Report, $n^{\circ} 250$, J une 2014 ; Gas for China, Energy E conomist, $n^{\circ} 392$, J une 2014 ; China Deal Makes W aves for LNG Shippers, Petroleum Intelligence Weekly, 2 J une 2014 ; Henderson (2014) ; Cornot-Gandol phe (2014).

Pour l'heure, les exportations de gaz naturel de la Russie à partir du Power of Siberia restent limitées, même si elles sont susceptibles d'être portées à $60 \mathrm{Gm}^{3}$. Elles pourraient également être complétées par la mise en service d'un second gazoduc ouvrant une route d'exportation par l'ouest à partir des gisements de Sibérie occidentale (le projet A ltaï). La Chine pourrait dès lors se positionner comme une voie de diversification majeure des exportations gazières russes. Enfin, notons que la crédibilité de la politique de GNL russe sera renforcée par la réalisation du Power of Siberia, dès lors que ce dernier permet d'améliorer la rentabilité de certains projets de GNL. II permettra en effet d'alimenter en gaz les projets de GNL à partir de Vladivostok où serait construite une usine de liquéfaction permettant d'approvisionner d'autres grands consommateurs de la zone, notamment le J apon et la Corée ${ }^{12}$.

Ces évolutions restent toutefois soumises à un certain nombre d'incertitudes, notamment celles relatives à l'évolution de l'offre et de la demande gazières chinoises. Celles-ci conditionneront le niveau des importations gazières de la Chine, dont les estimations varient entre 90 et $170 \mathrm{Gm}^{3}$ en 2020 (Cornot-Gandol phe, 2014 ; Gao, 2012). De ce point de vue, deux facteurs méritent une attention particulière. $D^{\prime}$ une part, les augmentations de prix, suite aux mouvements de réformes des dernières années (Chen, 2014), sont susceptibles d'impacter significativement et de manière durable la demande gazière chinoise. D'autre part, le développement des gaz non conventionnels, un objectif prioritaire du gouvernement, pourrait accroître significativement $I^{\prime}$ offre chinoise. $M$ ais en la matière, plus que sur les réserves ${ }^{13}$, les inconnues portent sur le niveau de rentabilité des gaz non conventionnels (fonction des coûts de production mais aussi du niveau de prix du gaz naturel en Chine et de la capacité du gouvernement à mettre en place un cadre institutionnel, juridique et fiscal adapté aux contraintes de ce dével oppement (Gao, 2012 ; Chou, 2013 ; G unningham, 2013) ${ }^{14}$.

\section{$2.2 \mathrm{~L}$ a question des structures de gouvernance}

A u-delà des incertitudes existantes de part et d'autre, le développement des échanges gaziers entre la Russie et la Chine peut être facilité par une certaine cohérence institutionnelle des industries gazières. Cette cohérence institutionnelle au sens de la théorie néo-institutionnaliste s'exprime dans des modes organisationnels et plus largement dans des structures de gouvernance relativement semblables. Celles-ci répondent aux spécificités institutionnelles de leur environnement (cf. tableau 3). De ce point de vue, la question de l'alignement entre la

\footnotetext{
${ }^{12}$ La production de gaz naturel à partir de ce gisement devrait débuter en 2016, avec une production qui pourrait culminer à $25 \mathrm{G} \mathrm{m}^{3} /$ an (International G as Report, November 5, 2012).

${ }^{13}$ Selon I'US Energy Information Administration (2013), les ressources techniquement récupérables de shale gas de la Chine s'élèveraient à $311200 \mathrm{Gm}^{3}$. A cela s'ajouteraient des réserves significatives de gaz de houille ( $3^{\mathrm{e}}$ rang mondial derrière la Russie et le Canada) et des réserves de gaz de réservoir compact. $M$ ais ces estimations sont à prendre avec beaucoup de précautions.
}

${ }^{14} \mathrm{G}$ ao (2012) estime qu'en 2020 le niveau de production des shale gas de la Chine pourrait se limiter à $10 \mathrm{Gm}^{3}$. 
structure de gouvernance et I'environnement institutionnel (W illiamson, 2005 ; B rousseau et al., 2011) justifie des modes d'organisation et des structures de gouvernance des industries gazières relativement différenciés selon les environnements institutionnels considérés. II importe en effet de définir une cohérence entre structure de gouvernance, modèle organisationnel et environnement institutionnel dans les industries des hydrocarbures. Une double cohérence est à définir : la cohérence entre la matrice institutionnelle et la structure de gouvernance d'une part; de la cohérence entre la structure de gouvernance et les attributs de la transaction, d'autre part. De ce point de vue, les industries gazières, industries de rente, de ressources non renouvelables mais aussi industries de réseau, sont marquées par une spécificité élevée des actifs, non redéployables en matière de transport, et par une incomplétude en matière de droits de propriété dès lors que des droits de propriété publics sur les ressources en terre sont maintenus.

Les marchés gaziers en Chine comme en Russie sont des marchés que I'on peut qualifier de « duaux » au sens où ils sont dominés par une gouvernance de type hiérarchique adossée à des franges concurrentielles sur des segments définis du marché. Cette structure de gouvernance s'appuie d'abord sur des compagnies majoritairement détenues par I'Etat, Gazprom, Rosneft du côté russe, la CNPC, Sinopec du côté chinois, compagnies par ailleurs verticalement intégrées. Ces compagnies d'Etat répondent aux spécificités des environnements institutionnels russes et chinois, bas prix de l'énergie, incertitudes sur les droits de propriété, faiblesse du contrat et de certaines institutions de marché comme la fiscalité. En ce sens, elles se positionnent comme un complément/substitut à une régulation contractuelle (Locatelli \& Rossiaud, 2011). Les marchés gaziers sont ensuite marqués par une double logique des prix, celle des prix administrés maintenus à un bas niveau (notamment pour le secteur résidentiel ou certaines industries jugées prioritaires comme les engrais en Chine) et des prix libres, fonction d'un certain degré de concurrence sur d'autres segments. Les réformes de prix menées en Russie (Locatelli, 2014) et en Chine (Chen, 2014) visent progressivement à faire converger ces deux types de formation de prix, avec pour conséquence de faire sensiblement augmenter les prix du gaz en interne. $M$ ais ces deux logiques subsistent encore pour une partie importante des marchés gaziers russe et chinois. La différenciation des marchés avec l'émergence de segments plus concurrentiels s'affirme de part et d'autre comme la voie de réforme choisie pour accroître l'efficience des industries gazières.

Cette voie de réforme s'oppose clairement au modèle concurrentiel développé par l'UE à la place de mécanismes de coordination hiérarchiques et administratifs (Glachant \& Perez, 2007). Ce modèle est basé sur une dé-intégration verticale des industries gazières, un accès des tiers au réseau et des marchés adossés à des transactions spot et de court terme (cf. tableau 3). L'objectif est aussi de créer un marché unique du gaz naturel à l'échelle de I'UE.

Tableau 3 : M odèles organisationnels et système de gouvernance

\begin{tabular}{|c|c|c|}
\hline UE & Russie & Chine \\
\hline M arché concurrentiel & M arché dual & M arché dual \\
\hline $\begin{array}{l}\text { - Dé-intégration verticale } \\
\text { (unbundling) }\end{array}$ & - Intégration verticale & - Intégration verticale \\
\hline - Accès aux tiers pour les gazoducs & $\begin{array}{l}\text { - A ccès aux tiers mais } \\
\text { partiellement mis en œuvre } \\
\text { - Prix réglementés et prix libres sur }\end{array}$ & $\begin{array}{l}\text { - Pas d'accès aux tiers } \\
\text { - Prix réglementés et prix libres sur }\end{array}$ \\
\hline
\end{tabular}




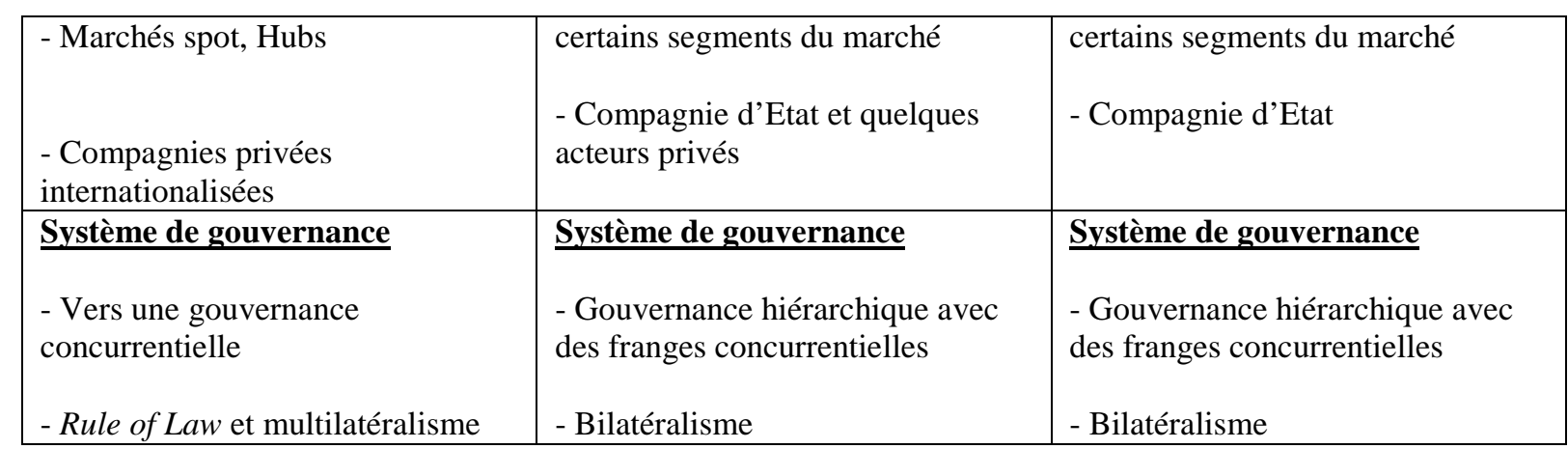

Sources : L ocatelli (2013) ; Shadrina (2014b)

Dans ces conditions institutionnelles, la gestion de la sécurité, objectif prioritaire des politiques énergétiques de l'UE, de la Russie et de la Chine, obéit à des logiques différenciées entre I'UE d'une part, et la Chine et la Russie d'autre part. On pourrait considérer que les enjeux pour I'UE et la Chine sont relativement semblables, puisqu'il s'agit de diversifier les fournisseurs pour obtenir une fourniture sécurisée et à un «prix acceptable ». L'enjeu est donc bien de limiter, voire d'exclure, tout pouvoir de marché de la part d'un fournisseur. La Russie à l'inverse, comme tout producteur, cherche avant tout à sécuriser sa demande tout en maximisant ses revenus. M ais au-delà de ce constat, les outils mis au service de cet objectif sont sensiblement différents entre I'UE d'un côté, la Chine et la R ussie de l'autre.

Pour l'UE, les institutions de marché, la Rule of law dans le cadre d'une conception multilatérale des relations internationales, sont censées produire de la stabilité et de la sécurité. Elles constituent les éléments déterminants de la structure de gouvernance du modèle concurrentiel des industries de réseau mis en place par I'UE. Dès lors, la gestion de la sécurité énergétique de l'UE, et notamment sa relation avec ses fournisseurs, est principalement basée sur la création d'un grand marché de l'énergie au travers de l'exportation des acquis communautaires (M cGowan, 2007). Le principal objectif est ainsi d'établir, notamment avec ses fournisseurs extérieurs, un espace commun de régulation au travers de règles susceptibles de normer le comportement des acteurs. La relation UE-Russie concernant les échanges de gaz naturel bute sur cette question. La non-ratification du traité de la Charte de l'E nergie est l'expression du refus de l'Etat russe de considérer les normes et règles de la structure de gouvernance concurrentielle de l'UE comme le cadre de régulation des échanges en matière de gaz naturel (Cameron, 2010) et plus largement comme outil de gestion de la sécurité énergétique.

Pour la Chine comme pour la Russie, la gestion de la sécurité énergétique ne peut se concevoir en dehors de l'Etat, notamment au travers de la mise en œuvre d'une diplomatie énergétique. A l'opposé du multilatéralisme promu par I'UE, les relations bilatérales entre Etats et entre companies sont dès lors privilégiées (Shadrina, 2014b; Vivoda, 2014). Les contrats de long terme adossés à des financements, les politiques de descente en aval ou d'accès à la ressource des companies énergétiques, sont partie prenante de cette logique. Ainsi, l'accord entre la Russie et la Chine ne se limite pas à des échanges de gaz naturel. Comme pour les accords pétroliers, il inclut des financements et probablement un accès aux ressources en hydrocarbures de la Russie. $M$ ais celui-ci se fait sous le contrôle (et avec l'aval) de l'Etat russe. Par ailleurs, les compagnies énergétiques russes sont en mesure de mener une stratégie de descente en aval sur le marché chinois (Shadrina \& Bradshaw, 2013), alors que dans le même temps les règles du troisième paquet Energie élaboré par I'UE contraignent 
fortement Gazprom dans la poursuite de cette stratégie pourtant prioritaire (Boussena \& Locatelli, 2013).

Tableau 4 : L es enjeux de politique énergétique

\begin{tabular}{|c|c|c|}
\hline UE & Russie & Chine \\
\hline $\begin{array}{l}\text { Objectif de la politique } \\
\text { énergétique }\end{array}$ & $\begin{array}{l}\text { Objectif de la politique } \\
\text { énergétique }\end{array}$ & $\begin{array}{l}\text { Objectif de la politique } \\
\text { énergétique }\end{array}$ \\
\hline $\begin{array}{l}\text { Sécuriser l'approvisionnement par } \\
\text { la diversification des fournisseurs }\end{array}$ & $\begin{array}{l}\text { Sécuriser la demande par la } \\
\text { diversification des marchés } \\
\text { d'exportation }\end{array}$ & $\begin{array}{l}\text { Sécuriser l'approvisionnement par } \\
\text { la diversification des fournisseurs }\end{array}$ \\
\hline Moyens & Moyens & M oyens \\
\hline $\begin{array}{l}\text { La concurrence : transaction de } \\
\text { court terme }\end{array}$ & Contrat de long terme de type TOP & $\begin{array}{l}\text { Contrat de long terme de type TOP } \\
\text { et concurrence entre les } \\
\text { fournisseurs }\end{array}$ \\
\hline $\begin{array}{l}\text { A ccès aux ressources au travers } \\
\text { d'un cadre d'investissement } \\
\text { multilatéral }\end{array}$ & $\begin{array}{l}\text { Accès aux ressources sous le } \\
\text { contrôle de l'Etat }\end{array}$ & $\begin{array}{l}\text { A ccès aux ressources dans les pays } \\
\text { fournisseurs au travers des } \\
\text { companies d'Etat }\end{array}$ \\
\hline $\begin{array}{l}\text { Création d'un marché unique avec } \\
\text { les producteurs par I'exportation } \\
\text { des acquis communautaires }\end{array}$ & $\begin{array}{l}\text { Relations bilatérales d'E tat à E tat: } \\
\text { diplomatie énergétique }\end{array}$ & $\begin{array}{l}\text { Relations bilatérales d'E tat à E tat: } \\
\text { diplomatie énergétique }\end{array}$ \\
\hline $\begin{array}{l}\text { Compagnies privées } \\
\text { internationalisées }\end{array}$ & $\begin{array}{l}\text { Compagnies d'E tat } \\
\text { internationalisées }\end{array}$ & $\begin{array}{l}\text { Compagnies d'E tat } \\
\text { internationalisées }\end{array}$ \\
\hline
\end{tabular}

Le développement des échanges gaziers entre la Russie et l'A sie est une politique de long terme, la Chine étant de ce point de vue un marché essentiel. Cette évolution n'en demeure pas moins à court terme stratégique tant du point de vue de la Russie que de la Chine. Elle s'inscrit en effet dans une logique de sécurité énergétique par la diversification des marchés d'exportation pour la $R$ ussie et des fournisseurs pour la Chine. La proximité des marchés est un facteur important des logiques d'échange qui se mettent en place. M ais celles-ci pourraient être d'autant plus durables qu'elles s'insèrent dans un cadre institutionnel particulier marqué par des structures de gouvernance des industries gazières relativement cohérentes entre elles et fortement opposées au modèle concurrentiel de I'UE. Les modal ités de gestion de la sécurité énergétique en sont fortement impactées.

\section{REFERENCES BIBLIOGRAPHIQUES}

A OKI M . (2001), Toward a Comparative Institutional Analysis, Cambridge: M IT Press.

BOLTZ W. \& K ONOPLYANIK A. (2013), $7^{\text {th }}$ EU -Russia Gas Advisory Council M eeting: Internal Market, Progress report, B russel, 14 J une; http://www.konoplyanik.ru/speeches/GAC June_2013_B Bussels_WBO_AK_v6_merged final4.pdf

BOLTZ W. \& K ONOPLYANIK A. (2012), EU-Russia Energy Dialogue, Gas Advisory Council, Third Meeting: Workstream on Internal Market. Brussel, 24 A pril ; http://core.theenergyexchange.co.uk/agile_assets/1646/09.25_Representing_Russia_3.pdf

B OUSSENA S. \& LOCATELLI C. (2013), «Energy institutional and organisational changes in EU and Russia: Revisiting gas relations », Energy Policy, vol. 55, pp. 180-189. 
Brousseau e., Garrouste P. \& Raynaud E. (2011), "Institutional changes: Alternative theories and consequences for institutional design », Journal of Economic Behavior \& Organization, vol. 79, $n^{\circ}$ 1/2, pp. 3-19.

CAMERON F. (2010), "The politics of EU-Russia energy relations », in K. Talus \& P. Fratini, (eds), EU-Russia Energy Relations, Brussels: Euroconfidential, pp. 25-38.

CHEN M. (2014), The Development of Chinese Gas Pricing, Oxford Institute for Energy Studies, OIES Paper NG 89.

CHou E. (2013), Shale gas in China : D evelopment and challenges, Harvard University Law, draft paper.

CORNOT-GANDOLPHE S. (2014), Stratégie gazière de la Chine: développer la concurrence entre production nationale et importations, Paris : Ifri, N ote du centre Energie, octobre, $89 \mathrm{p}$.

DIXIT A. (2009), «Governance Institutions and Economic A ctivity », American Economic Review, vol. 99, nº 1, pp. 5-24.

GAO F. (2012), Will There Be a Shale Gas Revolution in China by 2020 ? Oxford Institute for Energy Studies, OIES paper NG 61.

GLACHANT J. \& PEREZ Y. (2007), Institutional Economics and Network, Industry Deregulation Policy. Groupe Réseaux J ean M onnet, U niversité de Paris-Sud 11.

GunNinghaM N. (2013), «A Shale gas revolution for China? », Climate policy, vol. 14, n², pp. 302-320.

Hautecloque De A.\& GLachant J.M. (2011), «Long-term contracts and competition policy in European energy markets», in J.M. Glachant, D. Finon, \& A. de Hautecloque, eds, Competition, Contracts and Electricity M arkets: A new perspective, Cheltenham : Edward Elgar, pp. 201-232.

Henderson J. (2014), The Commercial and Political Logic for the Altai Pipeline, Oxford Institute for Energy Studies, Oxford Energy Comment, December.

HENDERSON J. \& STERn J. (2014), The potential impact on Asia Gas Markets of Russia's Eastern Gas Strategy, Oxford Institute for Energy Studies, Oxford Energy Comment, February.

Henderson J. \& Pirani S., eds (2014), The Russian Gas Matrix: How Markets Are Driving Change, Oxford University Press.

Henderson J. (2011), The Pricing Debate over Russian Gas Exports to China, Oxford Institute for Energy Studies, OIES paper NG 56.

K OMLEv S. (2013), Pricing the "Invisible" Commodity. M oscow: Gazprom Export. Contract Structuring and Pricing Directorate, Discussion Paper $n^{\circ} 11$.

K ONOPLYANIK A. (2005), «Russian Gas to Europe: From long term contracts, on border trade and destination clauses to...? " , J ournal of Energy \& Natural Resources Law, vol. 23, n 3, pp. 282-307.

LoCATElli C. (2014), "The Russian gas industry : challenges to the 'Gazprom model' ? ", Post Communist Economies, vol. 26, $n^{\circ} 1$, pp. 53-66.

LOCATELLI C. (2013), "EU-Russia trading relations: the challenges of a new gas architecture », European J ournal of Law and Energy, online N ovember.

LOCATELLI C., ROSSIAUD S. (2011), «A neo institutionalist interpretation of the changes in the Russian oil model », Energy Policy, vol. 39, n 9, pp. 5588-5597.

MCGOWAN F. (2007), "Can the European Union's market liberalism ensure security in a time of 'Economic nationalism'? », J ournal of Contemporary E uropean Research, vol. 4, n² 2, pp. 90-106.

M ITRova T. (2014), The Geopolitics of Russian Natural Gas, Center for Energy Studies, Rice University's Baker Institute, Harvard K ennedy School.

PAIK K. (2012), Sino-Russian Oil and Gas Cooperation : the reality and implications, Oxford U niversity Press.

PERCEBOIS J., (2008), "The supply of natural gas in the European Union - Strategic issues », OPEC Energy review, vol. 32, n 1, pp. 33-53.

Romanova T. (2014), "Russian energy in the EU market: Bolstered institutions and their effects », Energy Policy, vol. 74, pp. 44-53.

Shadrina E. (2014a), "Russia's Gas Policy in Asia: The Driving Forces and the Nature of Institutional Changes », International J ournal of Business and M anagement, vol. 2, $n^{\circ} 4$, pp. 60-78.

SHADRINA E. (2014b), "Russia's natural gas policy toward Northeast Asia: Rationales, objectives and institutions », Energy Policy, vol. 74, pp. 54-67.

SHADRINA E. \& BRADSHAW M. (2013), «Russia's energy governance transitions ans implications for enhanced cooperation with China, Japan, and South K orea », P ost Soviet Affairs, vol. 29, n 6, pp. 461-499. 
STERN J. (2014), "The impact of European Regulation and Policy on Russian Gas Exports and Pipelines », in J. Henderson \& S. Pirani, eds, The Russian Gas Matrix: How Markets are Driving Change, Oxford University Press, pp. 82-107.

STERn J. \& ROgERS H. (2013), The transition to hub based pricing in Continental Europe: A response to Sergei Komlev of Gazprom Export, Oxford Institute for Energy Studies, Oxford Energy Comment.

TALUS K. (2011), "Long-term natural gas contract and antitrust law in the European Union and the United States », J ournal of World Energy Law and Business, vol. 4, n 3, pp. 260-315.

US Energy Information Administration (2013), Technically Recoverable Shale Oil and Shale gas Resources: An Assessment of 137 Shale F ormation in 41 Countries 0 utside the U nited States, J une.

VIVODA V. (2014), «Natural gas in A sia: Trade, Markets and regional institutions », Energy Policy, vol. 74, pp. 80-90.

W ILliAm SON O. (2005), "The Economics of Governance », American Economic Review, vol. 95, n² 2, pp. 1-18.

WILLIAMSON 0. 2000), "The New Institutional Economics: Taking Stock, Looking A head ", Journal of Economic Literature, vol. 38, n 3, pp. 595-613.

Y AFIMAVA K. (2013), The EU Third Package for Gas and the Gas Target Model: major contentious issues inside and outside the EU, Oxford Institute for Energy Studies, OIES paper NG 75. 